\title{
Inhibiting miR-182-3p Alleviates Gestational Diabetes Mellitus by Improving Insulin Resistance in Skeletal Muscle
}

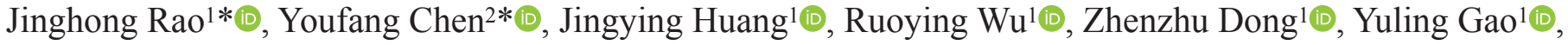 \\ Xuan Chen 1 (1)
}

${ }^{1}$ Department of Obstetrics and Gynecology Quanzhou First Hospital Affiliated to Fujian Medical University, Fuzhou, China

${ }^{2}$ Department of Clinical Medicine Quanzhou Medical College, Fuzhou, China

*They contributed equally to the article.

\begin{abstract}
Background: Gestational diabetes mellitus (GDM) is one of the most common metabolic diseases occurring during pregnancy. MiR-182$3 p$ participates in a variety of physiological processes such as cell proliferation, apoptosis, differentiation, and migration5, but its role in GDM is largely unknown.

Aims: To investigate the relationship between miRNA-182-3p and GDM and explore a potential therapeutic strategy for GDM.

Study Design: Animal experimentation

Methods: To evaluate the effect of miRNA182-3p in GDM, mice were separated as negative control (NC), miRNA-182-3p mimic or miRNA182-3p inhibitor, and miRNAs were administered intraperitoneally. Additionally, miRNA-182-3p mimic or miRNA-182-3p inhibitor was transfected into $\mathrm{C} 2 \mathrm{C} 12$ cells to evaluate glucose metabolism and insulin-related pathways.
\end{abstract}

Results: The miR-182-3p mimic accelerated GDM, which was effectively reversed by the inhibitor in GDM mice $(P=0.005$, miR$182-3 p$ inhibitor vs. mimic). Insulin receptor 1 (INSR1) was predicted to be the direct target gene of miR-182-3p using online tools. In addition, the miR-182-3p mimic inhibited INSR1 expression and insulin-related pathways in vivo and in vitro, which were all reversed by the miRNA82-3p inhibitor. Furthermore, the miR-182-3p mimic impaired glucose uptake and consumption by inhibiting translocation of glucose transporter type 4 (GLUT4) toward the $\mathrm{C} 2 \mathrm{C} 12$ cell membrane ( $P=0.007$ vs. control), while the inhibitor accelerated these processes ( $P=0.032$ vs. control; $P=0.005$, miRNA-182-3p inhibitor vs. mimic).

Conclusion: Inhibiting miR-182-3p effectively alleviated the development of GDM through INSR1, suggesting a potential therapeutic strategy for GDM.

\section{INTRODUCTION}

Gestational diabetes mellitus (GDM) is a condition in which a woman without diabetes develops glucose intolerance during the second or third trimester of pregnancy. GDM is a severe pregnancy syndrome, affecting 1-33\% of pregnant women depending on the population, screening strategy, diagnostic method, and diagnostic thresholds. Insufficient insulin secretion from pancreatic beta cells does not meet the increased demand for insulin during the third trimester of pregnancy. Similar to cardiovascular disease, obesity, and type 2 diabetes mellitus (T2DM), the number of pregnant women with GDM is growing rapidly. ${ }^{1,2}$ Untreated GDM causes severe harm to the mother and fetus. Excessive blood glucose levels lead to malformations, dystocia, miscarriage, eclampsia, premature birth, and polyhydramnios. The insulin sensitivity of normal pregnant women decreases by about $60 \%$ during pregnancy and at the same time, their basal glucose production increases by about $30 \%$ to ensure the energy supply to the fetus. However, the balance between upregulated glucose production and downregulated insulin sensitivity is broken in patients with GDM, and insulin sensitivity decreases significantly in insulin-sensitive tissues, such as fat, muscle, and liver, leading to an imbalance in energy metabolism. The normal insulin response is destroyed, glucose uptake by the muscle tissue is reduced under insulin-induced conditions, and GDM develops. ${ }^{3}$

Insulin resistance in skeletal muscle is related to many factors, including intramyocellular lipids, mitochondrial defects, the endocrine effects of adipokines, and inflammation. We focused on microRNA (miRNA). miRNA is a type of single-stranded small

Corresponding author: Xuan Chen, Department of Obstetrics and Gynecology Quanzhou First Hospital Affiliated to Fujian Medical University, Fuzhou, China e-mail: chenxuan@fjmu.edu.cn

Received: September 01, 2021 Accepted: November 19, 2021 Available Online Date: March, 14, 2022 • DOI: 10.4274/balkanmedj.galenos.2021.2021-8-140

Available at www.balkanmedicaljournal.org

ORCID iDs of the authors: J.R. 0000-0002-6275-0929; Y.C. 0000-0002-5908-2325; J.H. 0000-0003-1530-143; R.W. 0000-0001-6836-6568; Z.D. 0000-0002-3453-7545; Y.G. 0000-0003-3018-4122; X.C. 0000-0001-9268-5665.

Cite this article as:

Rao J, Chen Y, Huang J, Wu R, Dong Z, Gao Y, Chen X. Inhibiting miR-182-3p Alleviates Gestational Diabetes Mellitus by Improving Insulin Resistance in Skeletal Muscle. Balkan Med J.; 2022; 39(2):121-9.

Copyright@Author(s) - Available online at http://balkanmedicaljournal.org/ 
molecule non-coding RNA that participates in gene expression and regulation. Previous studies have reported that abnormal expression of miRNA is involved in many diseases, including T2DM. During pregnancy, placental-derived exosomes are enriched with a variety of miRNAs, and these miRNAs reach all parts of the body through the circulatory system to regulate metabolism in various tissues. The level of exosomes in pregnant GDM patients is significantly higher than that of normal pregnant women throughout pregnancy, and these exosomes abnormally express miRNAs, including miR$182-3 p .{ }^{4}$ MiR-182-3p is a widely reported miRNA that participates in a variety of physiological processes, including cell proliferation, apoptosis, differentiation, and migration ${ }^{5}$, but its role in GDM is largely unknown. Therefore, in this study, we investigated the function and occurrence of miR-182-3p during the development of GDM.

\section{MATERIALS AND METHODS}

\section{GDM Mouse Model}

C57BL/6J female mice (8-weeks-old) were purchased from Cyagen Biosciences Inc. (Suzhou, China). All animal procedures were approved by the Ethics Committee of Quanzhou First Hospital Affiliated to Fujian Medical University (2014PD/ $\mathrm{CX}$ ). The GDM mouse model was established as described previously. ${ }^{6}$ Briefly, female mice were fed a $45 \%$ kcal highfat-sucrose diet (Research Diets D12451) 6 weeks before mating to induce diabetes, which continued during mating and pregnancy. The early stage of the estrus cycle was determined by vaginal smear. Then, we put the female and a normal male mouse together. Mating was confirmed the next morning by the presence of a vaginal suppository, defined as the first pregnant day (GD0). There were five groups in total, including the normal group (normal pregnant mice), the GDM group (GDM mice), the NC group (GDM mice injected with nonsense miRNA), the GDM + miR-182-3p mimic group (GDM mice injected with a miR-182-3p mimic), and the GDM + miR$182-3 p$ inhibitor group (GDM mice injected with a miR-182$3 p$ inhibitor). Non-sense miRNA, the miR-182-3p mimic, and the miR-182-3p inhibitor were purchased from GenePharma (Shanghai, China), and $20 \mu \mathrm{g}$ of each in saline $(2.5 \mathrm{ml})$ was injected into the tail vein on the first and fourth day of each week beginning on GD 0 respectively. At the same time, the normal and GDM groups were injected with the same amount of saline. All mice were weighed on the morning of GD0, GD9, and GD18. After a 12-h overnight fast on GD 15, blood was collected from the tail, and fasting blood glucose levels were determined with a glucose meter. Fasting insulin levels were determined using an enzyme-linked immunosorbent assay kit (Cusabio, Wuhan, China) according to the manufacturer's instructions. The HOMA-IR (Homeostatic Model Assessment for Insulin Resistance) is an insulin resistance score. HOMA$\mathrm{IR}=$ fasting blood glucose $(\mathrm{mmol} / \mathrm{L}) \mathrm{x}$ fasting serum insulin (mU/1)/22.5. A high HOMA-IR value indicates low insulin sensitivity (insulin resistance), whereas a low HOMA-IR value indicates high insulin sensitivity.
Another set of normal pregnant mice was randomly divided into three groups with equal average body weight and injected with non-sense miRNA, miRNA-182-3p, or the miRNA-182-3p inhibitor twice per week. Their body weight was recorded on GD0, GD9, and GD18.

\section{Oral Glucose Tolerance Test (OGTT)}

Blood glucose was tested in all mice on GD15 after a 12-h fast, following oral administration of $2.0 \mathrm{~g} / \mathrm{kg}$ aqueous glucose solution. Then, the blood glucose levels were examined after 30, 60, 90, and 120 min respectively. Blood glucose levels were recorded, and the area under the curve was analyzed according to a previous method. $^{7}$

\section{Dual-luciferase Reporter Assay}

An online database (https://cm.jefferson.edu/rna22/Interactive) was employed to predict the potential miR-182-3p targets as described previously ${ }^{8}$, and to determine whether insulin receptor 1 (INSR1) was a direct target gene of miR-182-3p. Fragments of the synthetic INSR1 3' untranslated region (UTR) (INSR-WT) containing potential binding sites and mutated potential binding sites of the INSR1 3' UTR (INSR-Mu) were inserted into the pMIR-reporter plasmid using T4 DNA ligase after digestion with a restriction endonuclease. INSR-WT and INSR-Mut were co-transfected with the miR-182-3p mimic into HEK 293T cells (ATCC, Manassas, VA, USA) for $48 \mathrm{~h}$. The cells were harvested, lysed, and centrifuged for $10 \mathrm{~min}$. The supernatants were used to perform the luciferase assay using a luciferase assay kit (Promega, Madison, WI, USA) according to the manufacturer's instructions. Luciferase activity was defined as RLUs of Renilla firefly luciferase/RLU. ${ }^{8}$

\section{Transfection}

The mouse C2C12 muscle cell line (Chinese Academy of Sciences, Shanghai, China) was cultured in DMEM medium with $10 \%$ fetal bovine serum and transfected with the NC, the miR-182-3p mimic, and the miR-182-3p inhibitor using the Oligofectamine transfection reagent (Invitrogen, Waltham, MA, USA). The cells were collected for further studies. ${ }^{9}$

\section{Western Blot}

The treated cells were lysed in radioimmunoprecipitation buffer with a cocktail of protease inhibitors, and western blot was performed as described previously. ${ }^{10}$ The antibodies used are listed below:

GLUT4 (\#2213), phospho-Akt (\#8599), total-Akt (\#9272S), phospho-AS160 (Thr642) (\#4288), ERK1/2 (\#9101S), phosphoERK1/2 (\#9102S), and INSR-1 (\#2382S) were purchased from Cell Signaling Technology (Danvers, MA, USA) (1:1,000 dilution). Total-AS160 (\#07-741) was obtained from Upstate $(1: 1,000$ dilution, Thermo Fisher, Waltham, MA USA). GAPDH (ab8245) was purchased from Abcam (1:3,000, Cambridge, MA, USA). 


\section{Real-time Polymerase Chain Reaction (PCR)}

Total mRNA was extracted from the skeletal muscle of mice or treated cells, and qPCR was performed as described previously. ${ }^{11}$

\section{Glucose Consumption}

$\mathrm{C} 2 \mathrm{C} 12$ cells were seeded in a 24 -well plate. After transfection with non-sense miRNA, the miR-182-3p mimic, or the miRNA182-3p inhibitor for $24 \mathrm{~h}$, the medium was replaced with a low glucose medium containing $100 \mathrm{nM}$ insulin for $12 \mathrm{~h}$. The glucose concentrations in the culture medium were measured using a glucose assay kit. Glucose consumption = total glucose in blank wells - glucose in the culture medium. ${ }^{12}$

\section{Glucose Uptake}

Cells were seeded in 6-well plates. After transfection with nonsense miRNA, the miR-182-3p mimic, and the miRNA-182-3p inhibitor for $24 \mathrm{~h}$, glucose uptake was determined using 2-NBDG as described previously. ${ }^{12}$ Briefly, treated cells were digested with trypsin and suspended in phosphate-buffered saline (PBS) with $1 \mu \mathrm{M}$ insulin and $50 \mu \mathrm{M} 2-\mathrm{NBDG}$ for $30 \mathrm{~min}$ at $37^{\circ} \mathrm{C}$ in the dark. After washing twice with pre-chilled PBS, the cells in PBS were analyzed by flow cytometry (Becton Dickinson, Franklin Lakes, NJ, USA). The mean fluorescence intensity served as the 2-NBDG uptake measure on a per-cell basis.

\section{GLUT4 Translocation}

GLUT4 translocation toward the plasma membrane was measured using a plasma membrane lawn assay as described previously. ${ }^{13}$ Briefly, after transfection with non-sense miRNA, the miRNA-182-3p mimic, or the miRNA-182-3p inhibitor for $24 \mathrm{~h}$, the cells were starved in serum-free medium, and treated with insulin $(100 \mathrm{nM})$. The cells were lysed by ultrasound using an Astrason XL 2020 ultrasonic processor (Heat SystemsUltrasonic, Farmingdale, NY, USA). After blocking, the lawns were incubated with anti-GLUT4 antibody at room temperature for $45 \mathrm{~min}$ followed by application of the secondary antibody (A488 conjugated donkey anti-goat $\mathrm{IgG}$ ). The lawns were examined individually, and the fluorescence intensity of each cell was quantified using LSMS EXCITER-ZEN software (Zeiss Efficient Navigation, Carl Zeiss, White Plains, NY, USA) and shown as intensity per unit area. Data are presented as fold values relative to the control.

\section{Statistical Analysis}

The sample size was determined by a method described previously. ${ }^{1,2}$ According to this method, the power analysis showed that a sample size of at least six mice/group had a $90 \%$ power to detect a bodyweight change of $5 \mathrm{~g}$ on GD18, assuming a 5\% significance level. Considering 20\% attrition, eight mice in each group were needed. All data are expressed as mean \pm standard deviation. The normality of the distribution of numeric parameters was tested with the Shapiro-Wilk test. The statistical analysis was carried out using ANOVA followed by the least-significant difference test to compare three means or Tukey's Honestly Significant Difference test to compare six or more means. Repeated-measures ANOVA was also applied followed by the Bonferroni post-hoc test.

\section{RESULTS}

\section{The miR-182-3p Inhibitor Alleviates GDM in GDM Mice}

First, we examined the relative expression of circulating miR$182-3 p$ in the normal pregnant and GDM mice during different stages of pregnancy. The expression of miR-182-3p was gradually enhanced during pregnancy and reached its highest level on GD 18 in the GDM mice. Compared to normal mice, the GDM mice displayed a significant increase in miR-182-3p expression during middle (GD9) $(1.97 \pm 0.58, P=0.027)$ and late pregnancy (GD18) (3.02 $\pm 0.79, P=0.003)$ (Figure 1a).

To evaluate the role of miR-182-3p in GDM, we injected non-sense miRNA, the miR-182-3p mimic, or the miR-182-3p inhibitor into GDM mice, and monitored their body weight during pregnancy, which is an important indicator of GDM. The body weight of GDM mice increased significantly on GD 0 compared to the normal group $(22.4 \pm 1.1 \mathrm{~g}, P<0.001)$, confirming the occurrence of GDM (Figure 1b). Injecting the miR-182-3p mimic increased the body weight of GDM mice $(P<0.001)$ on GD 9, compared to the NC group, which decreased after injecting the miR-182-3p inhibitor $(P$ $=0.003)$. However, no difference was observed between the NC group and the GDM + miR-182-3p inhibitor group. The mice in all groups gained weight continually during pregnancy, but mice in the GDM and NC groups gained more weight than the normal mice by GD 18. Mice injected with miR-182-3p further upregulated their body weight $(P<0.001)$, which was reversed by the miR-182-3p inhibitor $(P=0.004)$. Unlike on GD 9 , the body weight of mice injected with miR-182-3p decreased significantly compared with the NC group $(P=0.006)$ (Figure $1 \mathrm{~b}, 1 \mathrm{c})$, suggesting that longterm treatment with the miR-182-3p inhibitor alleviated GDM.

To further investigate the role of miR-182-3p in GDM, we injected the miR-182-3p mimic and miR-182-3p inhibitor into normal pregnant mice and compared them to the normal group. Injecting miR-182-3p did not alter the body weight of pregnant mice, but mice injected with the miR-182-3p mimic gained significantly more weight than those in the normal group $(24.5 \pm 1.3 \mathrm{~g}, P=$ 0.036 at GD9; $29.3 \pm 1.5 \mathrm{~g}, P=0.007$ by GD18) (Figure 1d). These data further indicate that miR-182-3p accelerated the occurrence of GDM and that inhibiting miR-182-3p alleviated GDM in GDM mice.

\section{The miR-182-3p Inhibitor Improves Glucose and Insulin Tolerance in GDM Mice}

Next, we performed the OGTT to test glucose tolerance in the GDM mice on GD 15. After a glucose gavage, blood glucose levels were significantly upregulated in all GDM mice compared to that in the normal group, and the blood glucose level increased the most in mice injected with the miR-182-3p mimic $(P<0.001)$. In contrast, the miR-182-3p inhibitor downregulated the blood glucose levels in GDM mice $(P=0.024)$. After $2 \mathrm{~h}$ of glucose gavage, the blood 
glucose level in normal pregnant mice returned to normal, while the levels in all other GDM mice remained high (Figure 2a). We analyzed the overall area under the GTT curve, and the data showed that the miR-182-3p mimic aggravated glucose intolerance $(37.2 \pm 3.3 \mathrm{mM} \cdot \mathrm{h}, P<0.001)$, whereas the miR-182-3p inhibitor improved glucose intolerance $(21.6 \pm 2.5 \mathrm{mM} \cdot \mathrm{h}, P=0.044)$ in GDM mice (Figure 2b). In addition, the GDM mice displayed a higher fasting blood glucose level than that of the normal group $(15.5 \pm 3.1 \mathrm{mM} / \mathrm{L}, P<0.001)$, which is a symptom of GDM (Figure 2c). Not surprisingly, the miR-182-3p mimic induced higher blood glucose levels, which were reduced by the miR-182-3p inhibitor $(P=0.002)$ (Figure 2c). Taken together, these data suggest that the miR-182-3p inhibitor improved glucose intolerance in GDM mice. Furthermore, we also measured fasting insulin levels and analyzed the HOMA-IR, which were consistent with the pattern of fasting glucose levels (Figure 2d, 2e). The miR-182-3p mimic further increased fasting insulin levels $(0.82 \pm 0.11 \mu \mathrm{g} / \mathrm{L}, P=0.004)$ and HOMA-IR $(77.4 \pm 8.4, P<0.001)$, which were ameliorated by the miR-182-3p inhibitor. These results indicate that the miRNA-182$3 p$ inhibitor effectively improved insulin intolerance.

\section{INSR1 is a Direct Target of miR-182-3p}

We explored the miR-182-3p targets to further understand their role in GDM. First, we predicted the potential miR-182-3p targets in an online database as described previously ${ }^{8}$ and determined that INSR1 may be a target of miR-182-3p because it had potential binding sites in its 3'UTR with miR-182-3p (Figure 3a).

The dual-luciferase reporter gene assay was performed to verify whether INSR1 is a direct target gene of miR-182-3p. The luciferase assay showed that co-transfecting with miR-182-3p and INSR-WT inhibited the transcription of downstream genes $(0.49 \pm 0.07, P=$ $0.004)$, whereas the reporter gene was expressed normally when we co-transfected with miR-182-3p and INSR-Mut (Figure 3b). These results indicate that miR-182-3p directly binds to INSR1 mRNA to inhibit it.

\section{miR-182-3p Impairs Insulin Signaling in C2C12 cells}

We speculated that miR-182-3p may regulate insulin signaling pathways, as miR-182-3p directly targeted the insulin receptor. In our study, we focused on skeletal muscle. First, we transfected non-sense miRNA, the miR-182-3p mimic, or the miR-182-3p inhibitor into $\mathrm{C} 2 \mathrm{C} 12$ cells and examined INSR1 expression. The miR-182-3p mimic decreased INSR1 protein $(0.45 \pm 0.07, P$ $=0.006)$ and mRNA levels compared to the $\mathrm{NC}(0.43 \pm 0.10$, $P=0.005)$, which were induced by the miR-182-3p inhibitor $(P=0.004$ and 0.035$)$ (Figure 4a, 4b). Next, C2C12 cells were stimulated with insulin to activate downstream signaling pathways after 24-h of starvation. The cells were treated with a

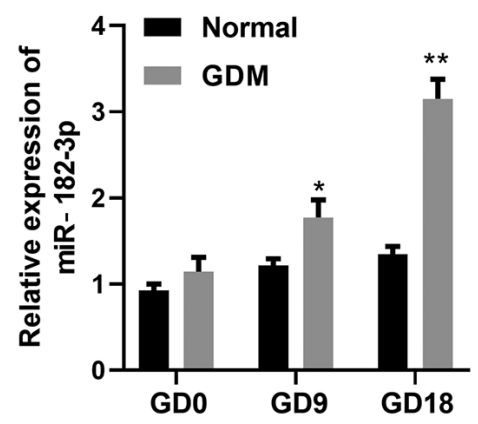

C

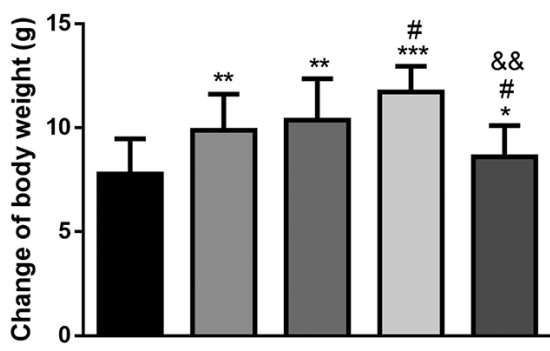

b

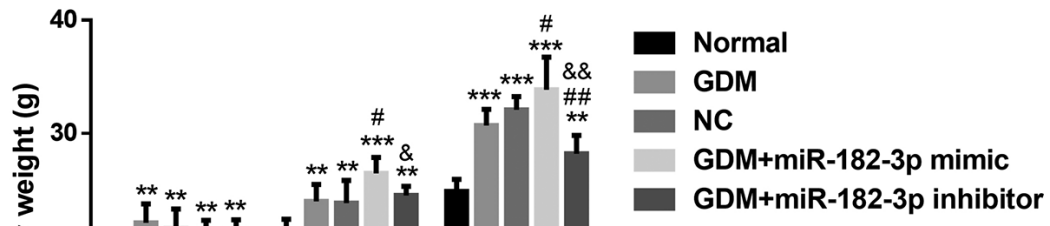

FIG. 1. The miR-182-3p inhibitor alleviated GDM in the GDM mice. (a) Relative expression of miR-182-3p in the normal pregnant group and the GDM group of mice during pregnancy. (b) Maternal body weight was recorded on gestational days (GD) 0, 9, and 18 in the normal group, the GDM group, the negative control (NC) group, the GDM+miR-182-3p mimic group, and the GDM+miR-182-3p inhibitor group. (c) Body weight changes were determined from GD0 to GD18. (d) The effect of miR-182-3p on normal pregnant mice was evaluated by monitoring maternal body weight; $n=8 / \mathrm{group}$. Data are presented as mean \pm SD. ${ }^{*} P<0.05,{ }^{* *} P<0.01,{ }^{* * *} P<0.001$ for comparison to the normal group. \#P<0.05, \#\#P<0.01 compared to the GDM group. $\& P<0.05, \& \& P<0.01$ compared to the miR-182-3p mimic group. GDM, gestational diabetes mellitus. 


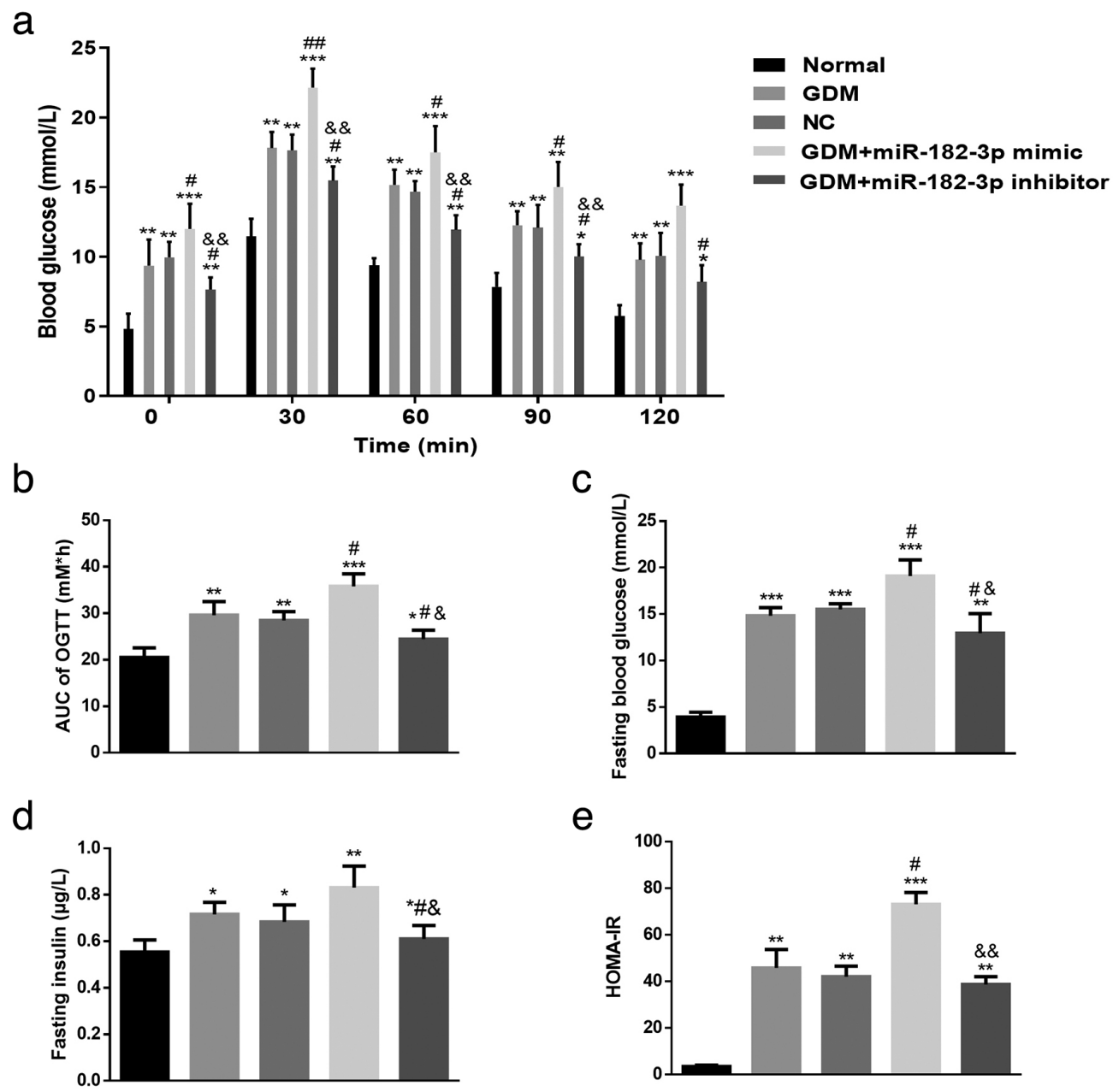

FIG. 2. The miR-182-3p inhibitor improved glucose tolerance in GDM mice. (a-b) Effect of miR-182-3p on glucose tolerance on GD 15. Blood glucose levels in mice of each group at different time points and the AUC for mice in each group. (b) Fasting glucose levels of the mice in each group; (c) Fasting insulin levels of the mice in each group; (d) HOMA-IR of the mice in each group. Data are presented as mean \pm SD. ${ }^{*} P<0.05,{ }^{* *} P<0.01$, ${ }^{* \star *} P<0.001$ for the comparison to the normal group. $\# P<0.05$, \#\#P<0.01 compared to the GDM group. \&P<0.05, \&\&P<0.01 compared to the miR-182-3p mimic group. GDM, gestational diabetes mellitus.

non-sense miRNA, the miR-182-3p mimic, or the miR-182-3p inhibitor for $24 \mathrm{~h}$. Insulin activated the p-Akt, p-AS160, and p-ERK1/2 pathways, and the miR-182-3p mimic deactivated these insulin-induced signaling pathways $(0.26 \pm 0.04, P<0.001$ for p-Akt; $0.18 \pm 0.03, P=0.003$ for p-AS160; $0.46 \pm 0.04, P=$ 0.002 for $p$-ERK1/2), which were reversed by the miR-182-3p inhibitor $(1.14 \pm 0.12, P=0.032$ for p-Akt; $1.26 \pm 0.21, P=$ 0.041 for $\mathrm{p}-\mathrm{AS} 160 ; 1.21 \pm 0.18, P=0.022$ for $\mathrm{p}$-ERK1/2) (Figure $4 \mathrm{c}-4 \mathrm{e})$. Taken together, miR-182-3p impaired the insulin-related signaling pathways in $\mathrm{C} 2 \mathrm{C} 12$ cells.

\section{miR-182-3p Inhibits the Insulin Signaling Pathway In Vivo}

To further confirm the inhibitory role of miR-182-3p on INSR1 and its downstream signaling pathways, we measured their expression in skeletal muscle of GDM mice. GDM reduced INSR1 protein expression $(0.39 \pm 0.04, P=0.003)$ and deactivated insulin-related pathways, including Akt $(0.28 \pm 0.05, P=0.003)$, AS160 $(0.31 \pm$
$0.06, P=0.004)$, and ERK1/2 $(0.52 \pm 0.05, P=0.006)$. All of these decreases were inhibited by the miR-182-3p mimic $(0.18 \pm 0.02$, $P=0.038$ for p-Akt; $0.27 \pm 0.03, P=0.019$ for $\mathrm{p}-\mathrm{AS} 160 ; 0.33 \pm$ $0.07, P=0.024$ for $\mathrm{p}$-ERK1/2, vs. GDM group), and induced by the miR-182-3p inhibitor $(0.32 \pm 0.04, P=0.027$ for p-Akt; $0.67 \pm$ $0.08, P=0.008$ for $\mathrm{p}-\mathrm{AS} 160 ; 0.65 \pm 0.10, P=0.042$ for $\mathrm{p}$-ERK $1 / 2$, vs. GDM group) (Figure 5a-5d). Therefore, miR-182-3p impaired the insulin signaling pathways in vivo and in vitro.

The miR-182-3p inhibitor enhances insulin-stimulated GLUT4 translocation, as well as glucose uptake and glucose consumption in $\mathrm{C} 2 \mathrm{C} 12$ cells.

Besides activating the downstream insulin pathways, INSR1 also regulates the translocation of glucose transporter type 4 (GLUT4) toward the plasma membrane. GLUT4 regulates glucose metabolism in skeletal muscle by facilitating glucose transport into 
INSR: 3'UTR: 5'-UAG T - - CAAGGUC CAGAACCA-3'

||||$\quad|||||||||| \mid$

miR-182-3p: 3'-AUCAACCGUUCAGAUCUUGGU-5'

b

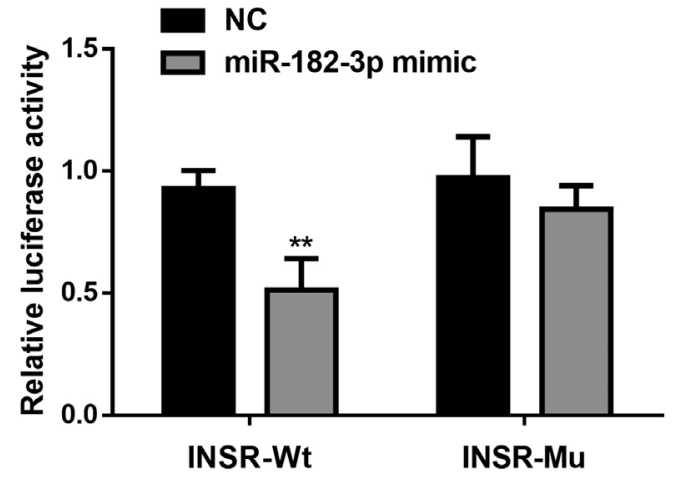

FIG. 3. INSR1 is a direct target gene of miR-182-3p. (a) The binding site sequences of INSR1 mRNA and miR-182-3p on the 3'-UTR. (b) Results of the dual-luciferase reporter assay. NC, negative control. Data are presented as mean $\pm \mathrm{SD}$ of at least three independent experiments. ${ }^{* \star} P$ $<0.01$ compared to the NC group. cells when insulin binds to its ligands and activates downstream pathways. We discovered that GLUT expression was not altered by either the miR-182-3p mimic or the inhibitor (Figure 6a). However, the miR-182-3p mimic significantly inhibited translocation of GLUT4 toward the plasma membrane $(0.22 \pm 0.03, P=0.003)$, which was markedly reversed by the miR-182-3p inhibitor (1.25 \pm $0.21, P<0.001$ vs. miR-182-3p mimic group) (Figure $6 \mathrm{~b}$ ) in $\mathrm{C} 2 \mathrm{C} 12$ cells. Consequently, glucose uptake $(0.43 \pm 0.06, P=0.003)$ and glucose consumption $(0.47 \pm 0.09, P=0.003)$ also decreased in response to miR-182-3p, which were both reversed by the miR$182-3$ inhibitor in $\mathrm{C} 2 \mathrm{C} 12$ cells $(1.31 \pm 0.05, P=0.029$ for glucose uptake; $1.27 \pm 0.11, P=0.005$ for glucose consumption) (Figure $6 \mathrm{c}, 6 \mathrm{~d})$. These data demonstrate that miR-192-3p regulates glucose metabolism through GLUT4 in skeletal muscle.

\section{DISCUSSION}

This study investigated the role of miR-182-3p in GDM and discovered that the miR-182-3p inhibitor impaired GLUT4 translocation, glucose uptake, and glucose consumption by directly binding to INSR1 and inhibiting its expression as well as its downstream signaling pathways in skeletal muscle, including Akt, ERk1/2, and AS160. In contrast, the miR-182-3p inhibitor promoted GLUT4 translocation toward the plasma membrane, a

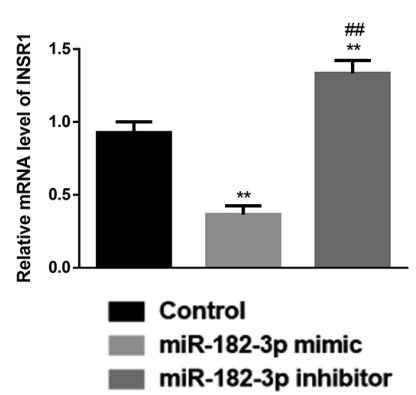

b

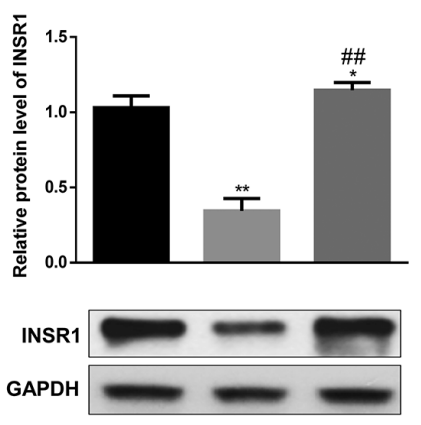

C

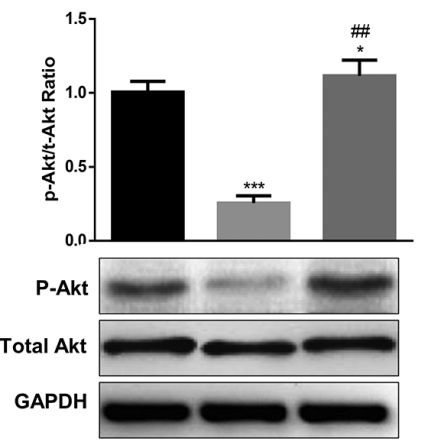

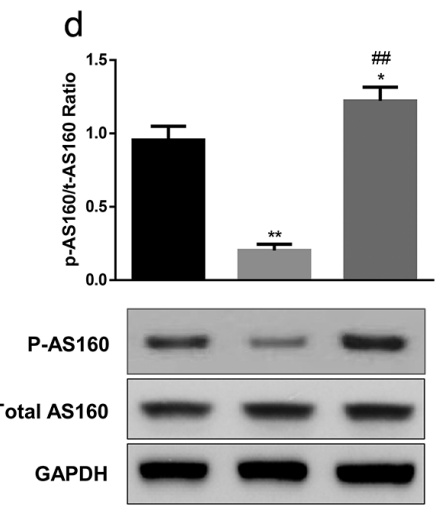

e

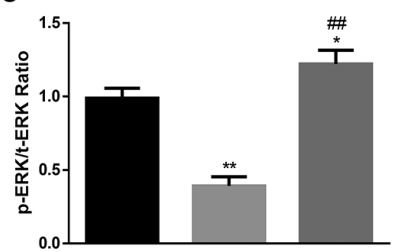

P-ERK1/2

Total ERK1/2

GAPDH

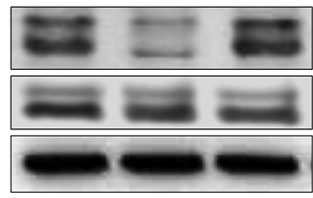

FIG. 4. miR-182-3p impairs the insulin signaling pathways in $\mathrm{C} 2 \mathrm{C} 12$ cells. (a, b) $\mathrm{C} 2 \mathrm{C} 12$ cells were treated with NC, the miR-182-3p mimic, or the inhibitor, respectively, and INSR1 expression was determined by qPCR and western blot. (c-e) Cells pre-treated with NC, the miR-182-3p mimic, or the inhibitor were stimulated with $100 \mathrm{nM}$ insulin after $24 \mathrm{~h}$ of serum starvation. Phosphorylated levels of Akt, AS160, and ERK1/2 were quantified by western blot. Corresponding representative western blots are shown. Data are presented as mean \pm SD of at least three independent experiments. ${ }^{*}$ $<0.05$, ${ }^{\star \star} P<0.01,{ }^{* \star} P<0.001$ for the comparison to the control. \#\#P<0.01 comparison between the miR-182-3p mimic group and the miR-182-3p inhibitor group. 


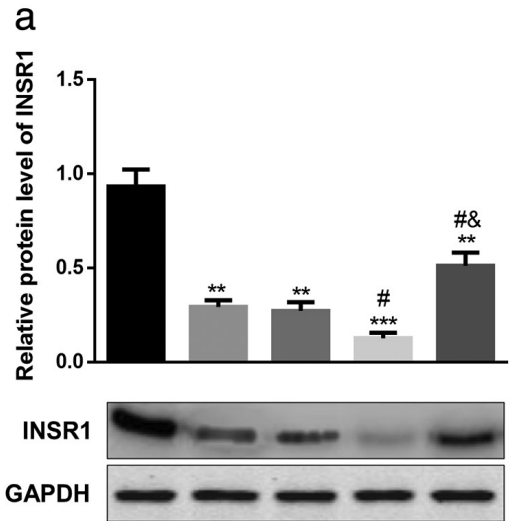

C
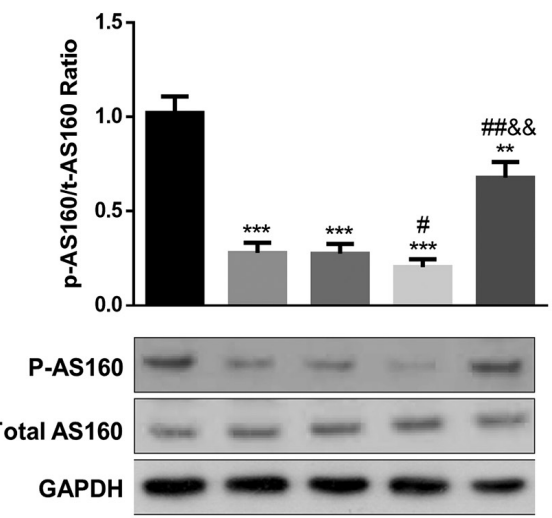

b

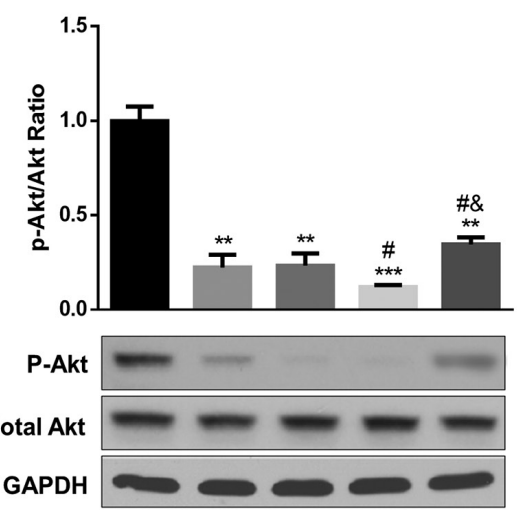

d

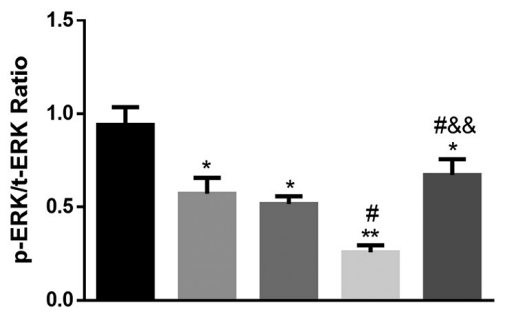

P-ERK1/2

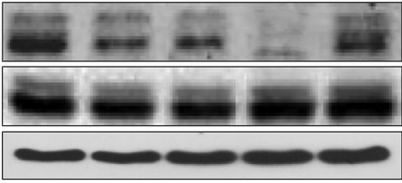

GAPDH

GDM+miR-182-3p inhibitor

FIG. 5. miR-182-3p impairs the insulin signaling pathways in vivo. Relative expression levels of INSR1 (a), phosphorylated levels of Akt (b), AS160 (c), and ERK1/2 (D) were quantified by western blot; $\mathrm{n}=8$ /group. Data are presented as mean \pm SD. ${ }^{*} P<0.05$, ${ }^{* \star} P<0.01,{ }^{* *} P<0.001$ for the comparison to the normal group. $\# P<0.05$, \#\#P<0.01 compared to the GDM group. \&P<0.05, \&\&P<0.01 compared to the miR-182-3p mimic group.

leading to upregulated glucose uptake and consumption by inducing INSR1 expression and activating insulin-stimulated signaling pathways in skeletal muscle. In addition, the miR-182$3 p$ inhibitor improved glucose and insulin tolerance in GDM mice and reduced their body weight, indicating that the miR-182-3p inhibitor alleviated the progression of GDM in the GDM mice. Our study suggests that inhibiting miR-182-3p may be a promising strategy for treating GDM.

MicroRNA is a type of single-stranded non-coding RNA that participates in a wide range of physiological and pathological processes. Therefore, microRNAs have received widespread attention. miR-182 participates in several diseases. For example, miR-182 suppresses apoptosis of cardiomyocytes in non-ischemic heart failure. ${ }^{14} \mathrm{miR}-182$ inhibits kidney fibrosis by mediating the transforming growth factor beta $1 / \mathrm{smad} 3$ signaling pathway. ${ }^{15}$ Moreover, miR-182 was discovered to be involved in the progression of several cancers, including ovarian carcinoma ${ }^{16}$, bladder cancer ${ }^{15,17}$, and non-small cell lung cancer. ${ }^{18,19}$ miR-182 is a promising biomarker of prostate cancer. ${ }^{20} \mathrm{miR}-182-5 \mathrm{p}$ accelerates the progression of preeclampsia. ${ }^{21}$ A relevant study demonstrated that miR-182 promotes cardiac function in diabetic mice. ${ }^{22}$ Although no study has investigated the relationship between miR182 and GDM, several miRNAs, including miR-182-3p, miR122-5p, miR-132-3p, and miR-1323 have been evaluated in GDM patients by a case-control study. ${ }^{4}$ That study reported that miR$182-3 p$ is differentially expressed in GDM patients, suggesting that miR-182-3p may be involved in GDM. Our current study determined for the first time that the miR-182 mimic accelerated GDM, and the miR-182 inhibitor effectively alleviated GDM.

GDM is the most common metabolic disease occurring during pregnancy and is caused by a decrease in maternal insulin sensitivity coupled with increased glucose production, leading to inadequate insulin response. Insulin resistance affects insulinmediated carbohydrate, lipid, and protein metabolism, and is reflected by the rate of downregulated glucose uptake into major insulin-sensitive tissues, such as skeletal muscle, liver, and adipose 

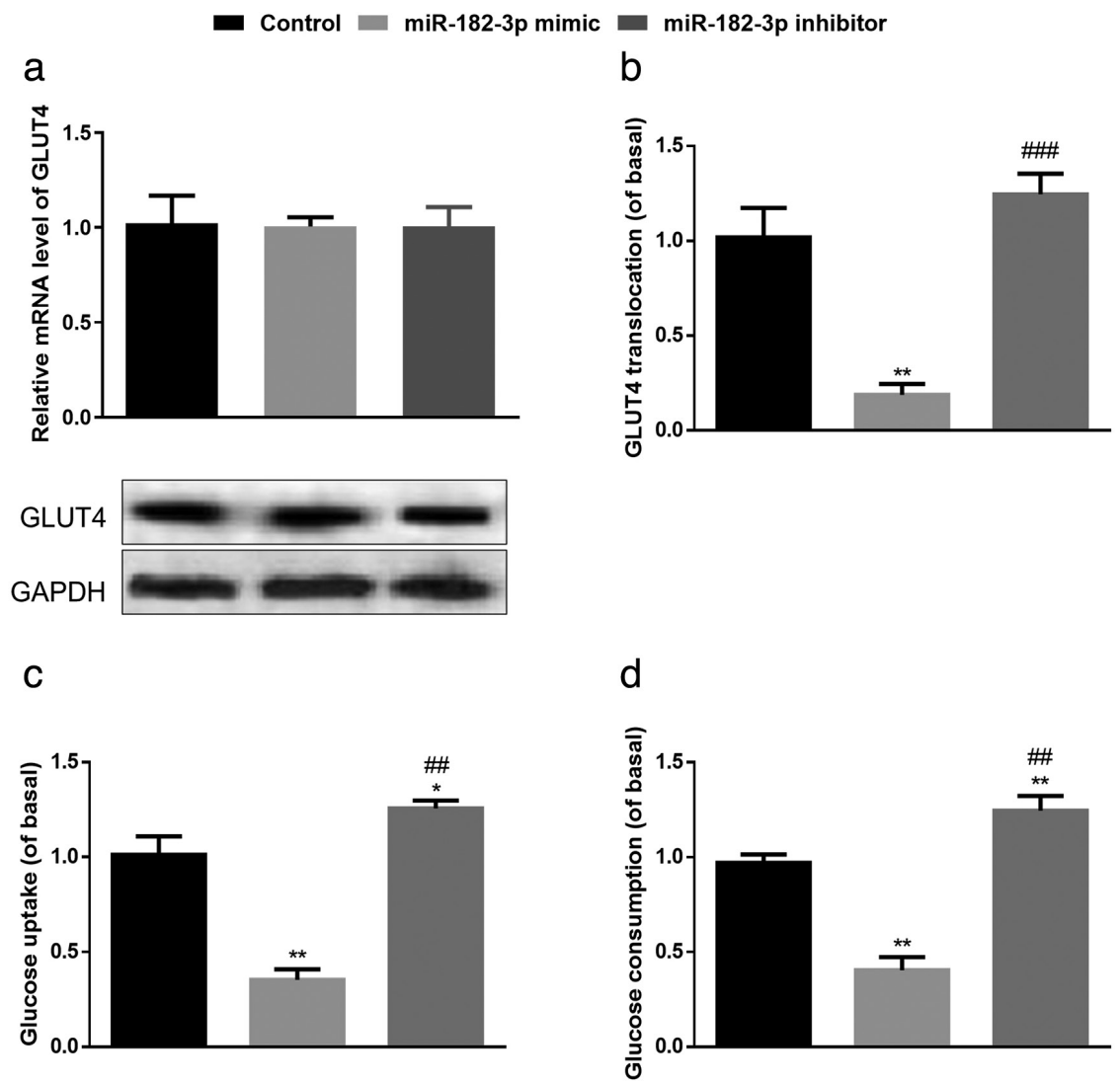

FIG. 6. The miR-182-3p inhibitor enhances insulin-stimulated GLUT4 translocation toward the plasma membrane, as well as glucose uptake and consumption in C2C12 cells. (a) Effects of miR-182-3p on GLUT4 mRNA levels and protein expression in C2C12 cells. (b) GLUT4 translocation toward the plasma membrane was measured using the plasma membrane lawn assay. (c, d) Effects of miR-182-3p on glucose uptake (c) and glucose consumption (d) in $\mathrm{C} 2 \mathrm{C} 12$ cells. Data are presented as mean $\pm \mathrm{SD}$ of six independent experiments. ${ }^{* *} P<0.01$ for comparison with control. \#\#P<0.01, $\# \# P<0.001$ between the miR-182-3p mimic group and the miR-182-3p inhibitor group.

tissue. ${ }^{23}$ Although it is still unknown whether insulin resistance occurs simultaneously in these insulin-sensitive tissues, our study targeted skeletal muscle, and we did not evaluate the role of miR$182-3 p$ in other tissues, such as liver or adipose tissue, which may need further investigation.

GDM is a hyperglycemic state that occurs in pregnant women and is a complex pathophysiological process. Chronic inflammation is the leading cause of GDM. However, in our current study, we did not investigate whether the miR-182-3p inhibitor alleviated the progression of GDM by inhibiting the inflammatory response. No study has reported that miR-182-3p affects inflammation. In addition, for the first time, our study discovered that INSR1 is a direct target of miR-182-3p. Due to the importance of insulin in the development of GDM, we only focused on INSR1 and its downstream signaling pathways in our study, and we did not study the relationship between miR-182-3p and other GDM pathogenic factors.

The insulin receptor has two substrates, such as INSR1 and INSR2. INSR1 and INSR2 both bind to insulin and insulin-like growth factor to activate separate downstream signaling pathways. Mice lacking the INSR1 or INSR2 gene can develop diabetes. Although INSR1 and INSR2 share a similar mechanism with insulin, they act differently. Unlike INRS1, INRS2 has been rarely investigated. Therefore, considering our limited laboratory conditions, we only focused on INSR1, which is the direct target of miR-182-3p predicted by an online database.

Unlike other GLUTs, GLUT4 is an insulin-mediated glucose transporter. ${ }^{5}$ GLUT4 takes up glucose from the bloodstream in skeletal muscle, the heart, and fat. ${ }^{24}$ Here, INSR1 was the direct target of miR-182-3p and miR-182-3p affected the insulin signaling pathways and glucose metabolism, which is why we only investigated the insulin-relevant glucose transporter GLUT4, and not the other GLUTs.

Taken together, we discovered that the miR-182-3p inhibitor promoted GLUT4 translocation, as well as glucose uptake and consumption by increasing the expression of INSR1 and its downstream signaling pathways in skeletal muscle. Therefore, the miR-182-3p inhibitor alleviates the development of GDM, suggesting that inhibiting the miR-182-3p inhibitor could serve as a potential strategy for treating GDM. 
A strength of this study was the discovery that miR-182-3p directly binds to INSR1, which is a key regulator of insulin-related pathways. Considering the important role of INSR1 in the insulin pathway, the miR-182-3p inhibitor may be a promising therapy for GDM. However, the miR-182-3p mechanism was only explored in $\mathrm{C} 2 \mathrm{C} 12$ cells, and we did not evaluate the effect of the miR-182-3p mimic or the miR-182-3p inhibitor in the muscle of GDM mice, which was a limitation. It will be necessary to evaluate the role of miR-182-3p in other insulin-related tissues, such as the liver, to further confirm the role of miR-182-3p in GDM.

In our study, we discovered that the miR-182-3p inhibitor promoted GLUT4 translocation, as well as glucose uptake and consumption by increasing the expression of INSR1 and its downstream signaling pathways in skeletal muscle. Therefore, the miR-182$3 p$ inhibitor alleviated the development of GDM, suggesting that inhibiting the miR-182-3p inhibitor may be a potential strategy for treating GDM.

Ethics Committee Approval: All the animal procedures were approved by the ethics committee of Quanzhou First Hospital Affiliated to Fujian Medical University.

Data Sharing Statement: The raw data supporting the conclusions of this article will be made available by the authors, without undue reservation.

Author Contributions: Design- X.C.; Data Collection or Processing- J.R., Y.C., J.H., R.W., Z.D., Y.G., X.C.; Writing- X.C.

Conflict of Interest: No conflict of interest was declared by the authors.

Funding: The study was supported by the Quanzhou science and technology plan project (2020C035R)

\section{REFERENCES}

1. Johns EC, Denison FC, Norman JE, Reynolds RM. Gestational Diabetes Mellitus: Mechanisms, Treatment, and Complications. Trends Endocrinol Metab. 2018;29:743754. [CrossRef]

2. Plows JF, Budin F, Andersson RA, et al. The Effects of Myo-Inositol and B and D Vitamin Supplementation in the $\mathrm{db} /+$ Mouse Model of Gestational Diabetes Mellitus. Nutrients. 2017;9:141. [CrossRef]

3. Catalano PM. Trying to understand gestational diabetes. Diabet Med. 2014;31:273281. [CrossRef]

4. Gillet V, Ouellet A, Stepanov Y, et al. miRNA Profiles in Extracellular Vesicles From Serum Early in Pregnancies Complicated by Gestational Diabetes Mellitus. J Clin Endocrinol Metab. 2019;104:5157-5169. [CrossRef]

5. Li J, Liu X, Li C, Wang W. miR-224-5p inhibits proliferation, migration, and invasion by targeting PIK3R3/AKT3 in uveal melanoma. J Cell Biochem. 2019;120:1241212421. [CrossRef]

6. Brawerman GM, Kereliuk SM, Brar N, et al. Maternal resveratrol administration protects against gestational diabetes-induced glucose intolerance and islet dysfunction in the rat offspring. J Physiol. 2019;597:4175-4192. [CrossRef]
7. Tang XW, Qin QX. miR-335-5p induces insulin resistance and pancreatic islet $\beta$-cell secretion in gestational diabetes mellitus mice through VASH1-mediated TGF- $\beta$ signaling pathway. J Cell Physiol. 2019;234:6654-6566. [CrossRef]

8. Chen SH, Liu XN, Peng Y. MicroRNA-351 eases insulin resistance and liver gluconeogenesis via the PI3K/AKT pathway by inhibiting FLOT2 in mice of gestational diabetes mellitus. J Cell Mol Med. 2019;23:5895-5906. [CrossRef]

9. Yu B, Liu Z, Fu Y, et al. CYLD Deubiquitinates Nicotinamide Adenine Dinucleotide Phosphate Oxidase 4 Contributing to Adventitial Remodeling. Arterioscler Thromb Vasc Biol. 2017;37:1698-1709. [CrossRef]

10. Lan S, Albinsson S. Regulation of IRS-1, insulin signaling and glucose uptake by miR-143/145 in vascular smooth muscle cells. Biochem Biophys Res Commun. 2020;529:119-125. [CrossRef]

11. Liu Z, Luo H, Zhang L, et al. Hyperhomocysteinemia exaggerates adventitial inflammation and angiotensin II-induced abdominal aortic aneurysm in mice. Circ Res. 2012;111:1261-1273. [CrossRef]

12. Sun B, Zhong Z, Wang F, et al. Atorvastatin impaired glucose metabolism in $\mathrm{C} 2 \mathrm{C} 12$ cells partly via inhibiting cholesterol-dependent glucose transporter 4 translocation. Biochem Pharmacol. 2018;150:108-119. [CrossRef]

13. Ishiki M, Nishida $Y$, Ishibashi $H$, et al. Impact of divergent effects of astaxanthin on insulin signaling in L6 cells. Endocrinology. 2013;154:2600-2612. [CrossRef]

14. Zhou F, Fu WD, Chen L. MiRNA-182 regulates the cardiomyocyte apoptosis in heart failure. Eur Rev Med Pharmacol Sci. 2019;23:4917-4923. [CrossRef]

15. Sun L, Hu C, Wang Z, Zhang X. MiR-182 inhibits kidney fibrosis by regulating transforming growth factor $\beta 1 / \mathrm{Smad} 3$ pathway in autosomal dominant polycystic kidney disease. IUBMB Life. 2020;72:1340-1348. [CrossRef]

16. Li Y, Li L. Prognostic values and prospective pathway signaling of MicroRNA-182 in ovarian cancer: a study based on gene expression omnibus (GEO) and bioinformatics analysis. J Ovarian Res. 2019;12:106. [CrossRef]

17. Carrera F, Andrade JC, Silva FJ, Simões J. Serum ferritin and hemochromatosis alleles in chronic hemodialysis patients. Nephron. 1988;50:196-198. [CrossRef]

18. Li Y, Zhang H, Gong H, et al. miR-182 suppresses invadopodia formation and metastasis in non-small cell lung cancer by targeting cortactin gene. J Exp Clin Cancer Res. 2018;37:141. [CrossRef]

19. Yang L, Dou Y, Sui Z, et al. Upregulated miRNA-182-5p expression in tumor tissue and peripheral blood samples from patients with non-small cell lung cancer is associated with downregulated Caspase 2 expression. Exp Ther Med. 2020;19:603610. [CrossRef]

20. Nayak B, Khan N, Garg H, et al. Role of miRNA-182 and miRNA-187 as potential biomarkers in prostate cancer and its correlation with the staging of prostate cancer. Int Braz J Urol. 2020;46:614-623. [CrossRef]

21. Fang YN, Huang ZL, Li H, et al. Highly expressed miR-182-5p can promote preeclampsia progression by degrading RND3 and inhibiting HTR-8/SVneo cell invasion. Eur Rev Med Pharmacol Sci. 2018;22:6583-6590. [CrossRef]

22. Meng Z, Wang Y, Lin Y, et al. [MicroRNA-182 modulates high glucose-induced cardiomyocyte hypertrophy via targeting Rac1]. Zhonghua Xin Xue Guan Bing Za Zhi. 2015;43:619-624. [CrossRef]

23. Catalano PM, Kirwan JP, Haugel-de Mouzon S, King J. Gestational diabetes and insulin resistance: role in short- and long-term implications for mother and fetus. $J$ Nutr. 2003;133(5 Suppl 2):1674s-1683s. [CrossRef]

24. Yaribeygi H, Farrokhi FR, Butler AE, Sahebkar A. Insulin resistance: Review of the underlying molecular mechanisms. J Cell Physiol. 2019;234:8152-8161. [CrossRef] 